\title{
Histochemical detection of the enzyme deficiency in blood films in Wolman's disease
}

\author{
BRIAN D. LAKE \\ From the Department of Morbid Anatomy, Institute of Child Health and Hospital for Sick Children, \\ Great Ormond Street, London
}

SYNOPSIS The clinical diagnosis of Wolman's disease (acid esterase deficiency) can be confirmed by a histochemical staining method using blood films. Acid esterase activity is found normally in lymphocytes, but in Wolman's disease only a very low level of activity can be detected. An intermediate level of activity was demonstrated in heterozygotes. The method may also be applicable in the prenatal detection of the disease.

The spectrum of clinical presentation of Wolman's disease (acid esterase/acid lipase deficiency) has been enlarged beyond the original descriptions (Abramov, Schorr, and Wolman, 1956; Crocker, Vawter, Neuhauser, and Rozowsky, 1965). Cases investigated by Lake and Patrick (1970), Young and Patrick (1970), and Orme (1970) may help to extend the definition to include the cholesterol ester storage disease of Fredrickson (1963) and Schiff, Schubert, McAdams, Spiegel, and O'Donnell (1968). This form of the disease may be relatively common since the condition is not debilitating and the patients may never have been seen by a physician.

The deficiency of acid esterase has been recognized in each type of presentation, and Young and Patrick (1970) have shown that the deficiency can also be demonstrated by assay of leucocyte preparations. A histochemical method to demonstrate the defect in blood films on slides is presented, and this should prove helpful in centres where specialist facilities for biochemical assays on leucocyte preparations are not available.

\section{Materials and Methods}

Blood films on slides (finger prick or sequestrene samples) were obtained from one case of the late infantile form of the disease, three cases of the juvenile form, siblings and parents of the affected juvenile cases, and a range of miscellaneous control subjects from whom blood had been taken for other purposes.

The films were stained by the May-Grunwald-
Geimsa sequence, and with oil red $O$ and sudan black (Lake, 1970) to demonstrate lipid deposits.

Acid esterase was demonstrated in blood films by a modification of the method described by Lake and Patrick (1970). Air-dried films were fixed for 10 minutes in formol-calcium acetate ( $4 \%$ formaldehyde in $0.1 \mathrm{M}$ calcium acetate) at room temperature. The slides, after a brief wash in tap water, were incubated at $37^{\circ} \mathrm{C}$ for four and a half hours in the following medium:

$a$-Naphthyl acetate. . . . Distilled water............25 ml

$0 \cdot 1 \mathrm{M}$ phosphate buffer $\mathrm{pH} 5 \cdot 0 \ldots 25 \mathrm{ml}$

Hexazonium pararosanline......

The $p \mathrm{H}$ was adjusted to $5 \cdot 8$ with $\mathrm{N}$. NaOH.

After incubation, the slides were washed in tap water, briefly counterstained (one minute) with Carrazzi's haematoxylin, alcohol dehydrated, and mounted, after clearing in xylene, in XAM (G. T. Gurr). A positive reaction is indicated by a reddish-brown colour.

The specificity of the reaction was checked by incubating some slides in the presence of $10^{-5} \mathrm{M}$ diethyl-paranitrophenyl phosphate (E600), which inhibits non-specific esterases. The lysosomal acid esterase is unaffected by this treatment (Lake and Patrick, 1970).

Some control blood films were left unfixed at room temperature for three and seven days before staining for acid esterase activity.

Electron microscopy was performed on buffy coat preparations after fixation in $4 \%$ glutaraldehyde, postfixation in $1 \%$ osmium tetroxide, and embedding in Araldite by conventional procedures. 


\section{Results}

Patients with acid esterase deficiency showed abnormalities in both large and small lymphocytes. Other cells appeared normal by each of the criteria adopted.

The cytoplasm of the lymphocytes was vacuolated and lipid deposits could be demonstrated within the vacuoles. The vacuolation and lipid deposits were more numerous in the infantile form of the disease.

Electron microscopy of the buffy coat preparations showed that the lipid-containing vacuoles in the lymphocytes were membrane-bound (Fig. 1), and therefore probably derived from lysosomes. No 'normal' lysosomes were seen in the lymphocytes, and other cellular organelles appeared normal.

In control films the lysosomal acid esterase activity, not inhibited by $10^{-5} \mathrm{M}$ E600, appeared as discrete droplets confined to the cytoplasm of lymphocytes (Fig. 2a). Slight, diffuse non-specific esterase activity, abolished in the presence of $10^{-5} \mathrm{M} \mathrm{E} 600$, was present in neutrophils. Intense diffuse activity was found in certain other cells (probably eosinophils) and this was also abolished by $10^{-5} \mathrm{M}$ E600. This activity in neutrophils and eosinophils was present in cases of acid esterase deficiency but in the lymphocytes the activity was very low or absent (Fig. 2b). In the severely affected case no activity could be detected in lymphocytes, but the older, milder cases showed? occasional lymphocytes with slight lysosomal activity

One hundred lymphocytes were counted in each $\vec{b}$ film and the percentage of those showing a positivereaction is recorded in Table $I$. No attempt wasw made to grade the intensity of the reaction product? Only those cells with no reaction product wereo counted as negative and all others, even though there was only a trace of activity, were counted as positive. $v$

The controls included a number of patients with leukaemia, and these showed a lower percentage of ${ }^{N}$ positive lymphocytes. If these are excluded the control range varies from $85 \%$ to $96 \%$ positive.

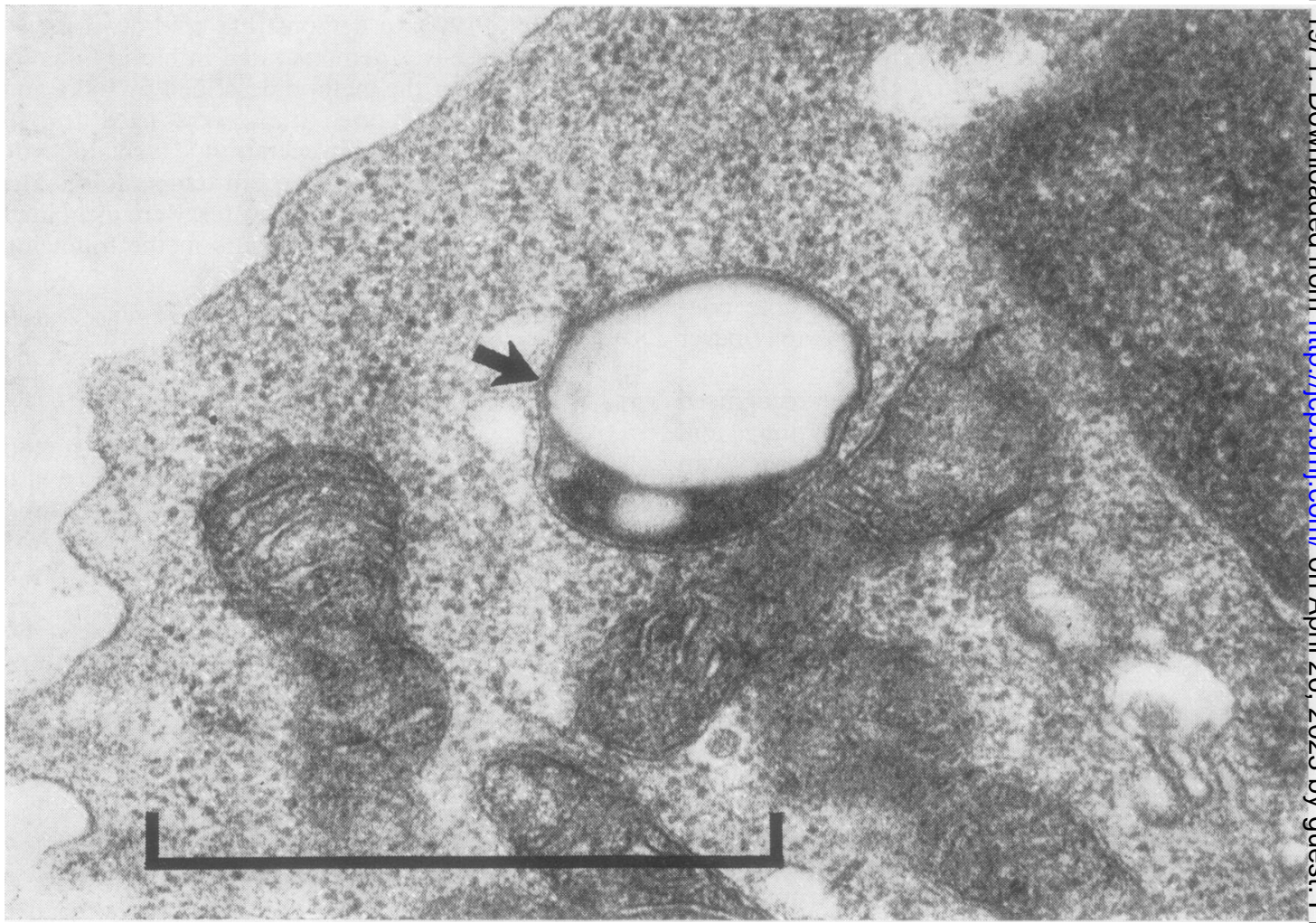

Fig. 1 Electron photomicrograph. Lymphocyte from patient with Wolman's disease. The membrane surrounding a lipid droplet is indicated by an arrow. Stained with uranyl acetate and lead citrate. Scale mark $1 \mathrm{~m} \mu$. 


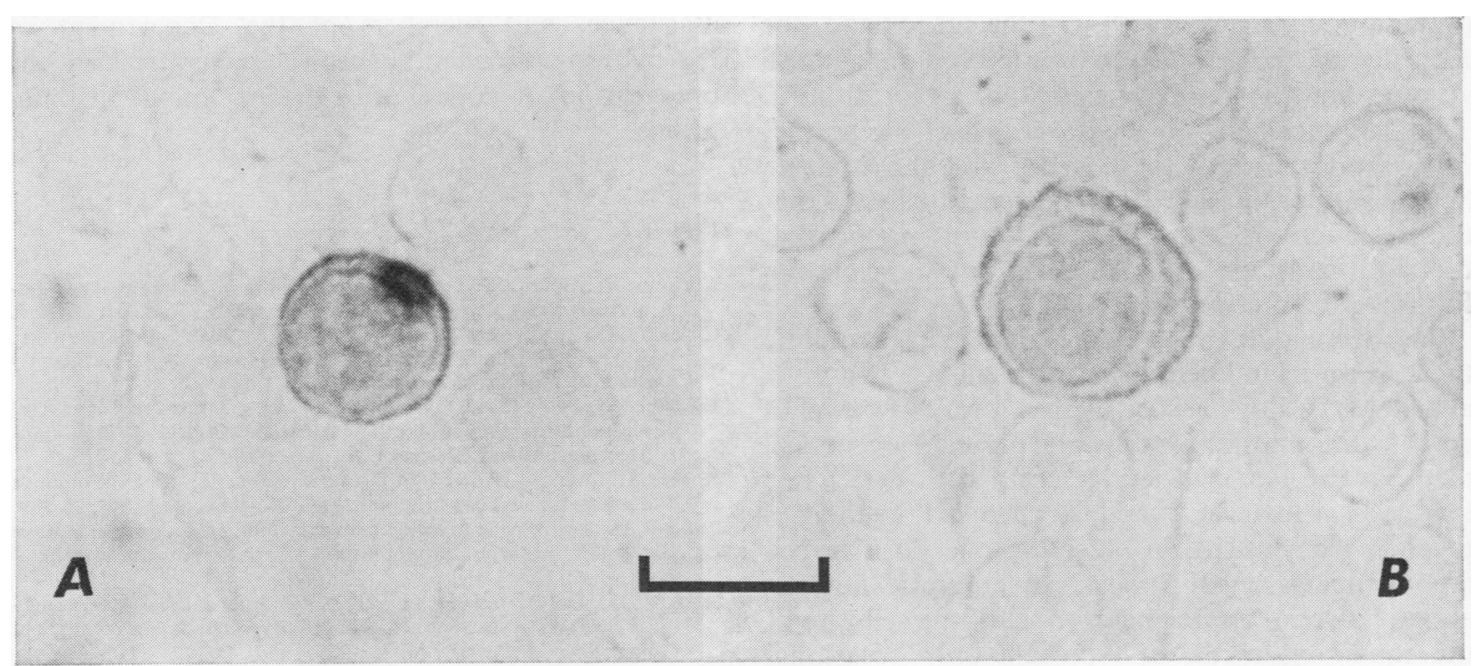

Fig. 2 Lymphocytes stained for acid esterase activity under identical conditions. $A$ normal control, $B$ Wolman's disease. Scale mark $10 \mathrm{m \mu}$

\begin{tabular}{ll}
\hline Cases & Percentage Lymphocytes Fositive \\
\hline Controls (33) & 87 (range 70-90) \\
Case 1 (B.R.) & 13 \\
Case 2 (W.R.) & 13 \\
Case 3 (A.R.) & 14 \\
Sib 1 (L.R.) & 75 \\
Sib 2 (B.R.) & 75 \\
Sib 3 (M.R.) & 86 \\
Father & 70 \\
Mother & 71 \\
Case 4 (A.B.) & 0 \\
\hline
\end{tabular}

Table I Percentage of lymphocytes positive

\section{Discussion}

The enzme deficiency in Wolman's disease, initially shown by a histochemical method to be that of an acid esterase (Lake and Patrick, 1970), causes accumulation of cholesteryl esters and triglycerides in lysosomes of cells of the reticuloendothelial system. The earlier described cases were all of the acute infantile type (Abramov et al, 1956; Wolman, Sterk, Gatt, and Frenkel, 1961; Crocker et al, 1965; Konno, Fujii, Watanuki, and Koizumi, 1966) but the cases with later onset have also been shown to be deficient in acid esterase/lipase (Young and Patrick, 1970). There is neither acid esterase activity (cholesteryl ester or p-nitrophenyl ester as substrate) nor acid lipase activity (triglyceride as substrate) in Wolman's disease (Patrick and Lake, 1969). It is therefore probable that these two enzymes are identical.
The juvenile cases of the disease (Orme, 1970; Lake, 1970; present series) which appear identical clinically and pathologically to cholesterol ester storage disease of Fredrickson (1963) and Schiff et $a l$ (1968) are also deficient in acid esterase/lipase activity (Young and Patrick, 1970). Diagnosis of all types of the disease can be made by assay of leucocyte preparations for acid esterase. The original histochemical method can also be applied to blood films and gives a reliable indication of the condition. The more acute cases show no activity in lymphocytes but the juvenile cases show some slight activity if the percentage of positively stained lymphocytes is calculated.

That some activity is found might indicate that there are isoenzymes of acid esterase, and that in the acute infantile type all isoenzymes are deficient; the juvenile form may only be deficient in one of the isoenzymes. This could account for the lack of severity of the symptoms and the relatively benign course.

The films left unfixed at room temperature before staining showed only minimal change from the original scores, and it would therefore seem possible to send unfixed blood films by post (1st class!) for confirmation of the diagnosis.

The activity recorded for obligate heterozygotes and for two of the patients was somewhat lower than the range of controls and it is possible that these percentages reflect the lower heterozygote level expected and found in parents of affected children 
(Young and Patrick, 1970). On the other hand, the intensity of the staining has not been taken into account and the results may be purely fortuituous. The difference between the lower end of the normal range and the activity recorded for the obligate heterozygotes is small. It would seem therefore that the detection of heterozygotes is not yet proved, though quantitation of the density of stain may in future be helpful in this respect.

The technique can also be applied to amniotic fluid. The prenatal detection of the disease is not yet available possibly because there is a very low level of activity in amniotic fluid cells, and present biochemical methods are not sufficiently sensitive to detect this low level. However, the histochemical method is much more sensitive in detecting one positive cell among many negative ones. In amniotic fluid at about 12 weeks of pregnancy most of the cells are of the squamous epithelial type and only occasional parabasal cells are present. The acid esterase stain is negative in squamous cells but is intensely positive in parabasal cells. This staining method may be the method of choice (coupled with electron microscopy) in the prenatal detection of Wolman's disease, until more sensitive biochemical methods are available.

I thank Miss J. Germain AIMLT for technical assistance, and members of the Department of Haematology for enthusiastic cooperation in theo preparation of coded samples for 'blind' examin ation.

\section{References}

Abramov, A., Schorr, S., and Wolman, M. (1956). Generalised xanthomatosis with calcified adrenals. Amer. J. Dis. Child.,91, 282-286. صి

Crocker, A. C., Vawter, G. F., Neuhauser, E. B. D., and Rosowsky, A. (1965). Wolman's disease: three new patients with a recently $\vec{\circ}$ described lipidosis. Pediatrics, 35, 627-640.

Fredrickson, D. S. (1963). Newly recognised disorders of cholesterol metabolism. (Presented at the American College of Physicians $\omega$ Meeting, Denver, Colorado.) Ann. intern. Med., 58, 718.

Konno, T., Fujii, M., Watanuki, T., and Koizumi, K. (1966). Wolman's disease: the first case in Japan. Tohoku J. exp. Med., 90, 375-389.

Lake, B. D. (1970). A histochemical evaluation of the glycogen storage diseases. A review of techniques and their limitations. Histochem. J., 2, 441-450.

Lake, B. D., and Patrick, A. D. (1970). Wolman's disease: deficiency of E600-resistant acid esterase activity with storage of lipids in lysosomes. J. Pediat., 76, 262-266.

Orme, R. L'E. (1970). Wolman's disease: an unusual presentation. Proc. roy. Soc. Med., 63, 489-490.

Patrick, A. D., and Lake, B. D. (1969). Deficiency of an acid lipase in $O$ Wolman's disease. Nature (Lond.), 222, 1067-1068.

Schiff, L., Schubert, W. K., McAdams, A. J., Spiegel, E. L., and $O$ O'Donnell, J. F. (1968). Hepatic cholesterol ester storage $\mathbb{\Phi}$ disease: a familial disorder. Amer. J. Med, 44, 538-546.

Wolman, M., Sterk, V. V., Gatt, S., and Frenkel, M. (1961). Primary $\vec{\varphi}$ familial xanthomatosis with involvement and calcification of the adrenals. Pediatrics, 28, 742-757.

Young, E. P., and Patrick, A. D. (1970). Deficiency of a cid esterase activity in Wolman's disease. Arch. Dis. Childh., 45, 664-668. 